\title{
Evaluation of different types of artificial intelligence methods to model the suspended sediment load in Tigris River
}

\author{
Mustafa Al-Mukhtar ${ }^{*}$ \\ ${ }^{1}$ Building and Construction Engineering Department. University of Technology, Baghdad
}

\begin{abstract}
Modeling of suspended sediment load in rivers has a major role in a proper management of water resources. Artificial intelligence has been identified as an efficient way to model the complex nonlinear hydrological relationship. In this study, Adaptive Neuro Fuzzy Inference System (ANFIS), in addition to two different kinds of Artificial Neural Network (ANN) i.e. feedforward and radial basis networks were used and compared to model the suspended sediment load (SSL) in Tigris River-Baghdad using the streamflow discharge as input. To this end, an intermittent data of SSL and streamflow were collected over the period 1962-1981 from Sarai station in Baghdad. $70 \%$ of these data was used to calibrate (train) the networks and the remaining $30 \%$ for the validation (test). The coefficient of determination $\left(\mathrm{R}^{2}\right)$, root mean square error (RMSE), and Nash and Sutcliffe model efficiency coefficient (NSE) were used to judge whether the observed and modelled data belong to the same distribution. Results revealed that the ANFIS model outperform the other methods. $\mathrm{R}^{2}$, RMSE, and NSE of ANFIS during the calibration phase were equal to $0.58,75617$, and 0.58 , respectively and during the validation were $0.72,27944$, and 0.59 , respectively. Therefore, ANFIS approach is recommended to estimate the river suspended sediment load.
\end{abstract}

\section{Introduction}

Modelling sediment transport accurately is of great importance tool in many fields of water resources management such as water quality, reservoir management, designing of dam and operation. It is well known that higher concentrations of suspended sediment are, the higher risky consequences on river ecology [1]. Therefore, it is of great importance to estimate the inflow amounts of sediment in water systems.

Artificial intelligence approaches such as artificial Neural Network (ANN), support vector regression, fuzzy logic (FL) are used in prediction of suspended sediment [2] as they provide a good alternative to conventional approaches like linear or deterministic models.

Artificial neural network (ANN) is a tremendously parallel- distributed information processing system, which is obtained from research on the nature of human brains [3]. Many advantages of this technique and in particular in the field of hydrology were proved. Foremost, it can manipulate with any arbitrary continuous functions, modeling a nonlinear system considering no prior assumption of processes involved, and ultimately can give a good solution when input data are incomplete or vague [4,5]. Recently, many successful applications of ANN's in the field of sediment transport were reported [6-15].

The Adaptive Neuro Fuzzy Inference System (ANFIS) is a kind of ANN that integrates both neural network and fuzzy logic principles [16]. It is characterized by the capability of capturing the benefits of both items in a single framework. In addition, the set of fuzzy IF-THEN rules that have learning capability to approximate nonlinear functions is adopted in its inference system [17]. Recently, many successful applications of ANFIS in the field of water resources were reported. For example, [18] applied the ANFIS model to modeling groundwater level fluctuation using hydro meteorological data (precipitation and temperature). They compared the results from the ANFIS model with multiple layer perceptron optimized by two different approach i.e. scaled conjugate gradient and Bayesian neural networks. They found that ANFIS outperform the other methods in modeling the noisefree hydro meteorological data. [19] used the ANFIS model to simulate the soil moisture (SM) in an arid area of a basin in China. Their results showed that ANFIS model could give accurate estimation of SM and can be successfully applied for SWC forecasting. [20] used the ANFIS model to estimate the suspended sediment concentration in Mad River Catchment near Arcata, USA. They compared results from ANFIS model with the generalized regression neural networks (GRNN), radial basis neural networks (RBNN) and multi-layer perceptron (MLP) and two different sediment-rating curves (SRC). They found that ANFIS model perform better than the other models in daily suspended sediment concentration estimation.

The objectives of this study is to investigate the capability of two types of artificial neural networks (i.e. 
feedforward and radial basis networks) and the ANFIS model to simulate instantaneous suspended sediment load in Sarai station-Baghdad using corresponding streamflow discharge as inputs. Therefore, three codes were prepared with the use of MATLAB software (Release 2008a). To the best of knowledge, no previous studies were conducted to simulate the suspended sediment load in Tigris River-Iraq using these approaches. The positive results from this study could be of use to provide a better insight for the stakeholder about the sediment load in Baghdad.

\section{Material and methods}

\subsection{Adaptive Neuro Fuzzy Inference System ANFIS}

ANFIS is a special type of ANNs which integrates both neural networks and fuzzy logic principles [21]. Thus, it considers a universal approximator as it has the capability of approximating any real continuous function on a compact set to any degree of accuracy using either Takagi-Sugeno or Mamdani fuzzy inference system (if-then rule) [22]. "Takagi and Sugeno's fuzzy if-then rules are characterized as the output of each rule is a linear combination of the input variables plus a constant term, and the final output is the weighted average of each rule's output" [21]. A hybrid-learning rule, which combines the gradient method and the least squares estimate to identify parameters pertaining membership function (MF), was adopted to be used in ANFIS since it is faster and not trapped by local minima as in the basic rule. As a typical example it is assumed a FIS has two parameters i.e. $x$ and $y$ and one output $f$. The first-order Sugeno's fuzzy model (a typical rule set with two fuzzy If-Then rules) might be written as:

Rule 1: If $x$ is $A_{1}$ and $y$ is $B_{1}$; then $f_{1}=p_{1} x+q_{1} y+$ $\mathrm{r}_{1}$

Rule 2: If $x$ is $A_{2}$ and $y$ is $B_{2}$; then $f_{2}=p_{2} x+q_{2} y+$ $\mathrm{r}_{2}$

Where $\mathrm{p}_{1}, \mathrm{q}_{1}, \mathrm{r}_{1}$ and $\mathrm{p}_{2}, \mathrm{q}_{2}, \mathrm{r}_{2}$ are the variables in the then-part (consequent part) of the first-order Sugeno fuzzy model. The general structure of ANFIS model is shown in Figure 2. A comprehensive details about ANFIS functioning could be found in [21]. In this study, each run with different numbers of member functions (MF) were tried and the one that gave the minimum squared error was selected. Triangular-shaped built-in MFs for the ANFIS models was found to be efficient for modelling SSL.

\subsection{Artificial neural networks ANN's}

\subsubsection{Feedforward neural network}

Feedforward neural network $(F F)$ is a multi-layer perceptron type in which nodes in one layer are only connected to nodes in the next layer, it has been used in many prediction subjects [23]. "Typically, feedforward networks have one or more hidden layers of sigmoid transfer function followed by an output layer of linear transfer function. Multiple layers of neurons with nonlinear transfer functions allow the network to learn nonlinear and linear relationships between input and output vectors. The linear output layer lets the network produce values outside the range -1 to +1 " [24]. A supervised learning technique called backpropagation is used to train the network. The backpropagation computation is derived using the chain rule of calculus [25]. The default algorithm used in optimizing weights between connections is Levenberg-Marquardt backpropagation [25].

\subsubsection{Radial basis neural network}

Radial basis network (RB) typically have three layers: an input layer, hidden layer with non-linear radial basis function (RBF), and linear output layer. The RBF estimates the output by using the standard Euclidean distance between the inputs and its corresponding weight [4, 24]. In other words, for each node the Euclidean distance between the center and the input vector is estimated, and then transformed by a nonlinear function (Exponential) that determines the output from the hidden layers. The radial basis function has a maximum of 1 when its input is 0 . When the distance between the weight (w) and input (p) decreases, the output increases. Thus, a radial basis neuron acts as a detector that produces 1 whenever the input $\mathbf{p}$ is identical to its weight vector $\mathbf{w}$.

\section{Model Assessment Criteria}

Three most widely used criteria were used in this study in order to test the validity of the evaluated models, which are:

i. Determination coefficient $\left(\mathrm{R}^{2}\right)$ : the determination coefficient (equation 3) "describes the proportion of the variance in observed data explained by the model. Its values range from 0 to 1 . Higher values indicate less error variance and typically values greater than 0.5 are considered acceptable" [26, 27].

$$
\mathbf{R}^{2}=\left[\frac{\sum_{\mathrm{i}=1}^{\mathrm{n}}\left(\mathbf{O}_{\mathrm{i}}-\overline{\mathbf{0}}\right)\left(\mathbf{P}_{\mathrm{i}}-\overline{\mathbf{P}}\right)}{\sqrt{\sum_{\mathrm{i}=1}^{\mathrm{n}}\left(\mathbf{O}_{\mathrm{i}}-\overline{\mathbf{0}}\right)^{2}} \sqrt{\sum_{\mathrm{i}=\mathbf{1}}^{\mathrm{n}}\left(\mathbf{P}_{\mathrm{i}}-\overline{\mathbf{P}}\right)^{2}}}\right]^{2}
$$

Where $\mathrm{O}_{\mathrm{i}}$ is the observed value, $\overline{\mathrm{O}}$ is the average observed value, $P_{i}$ is the predicted value, and $\bar{P}$ is the average predicted value.

ii. Root mean square error (RMSE): root mean square error (equation 4) is one of the error indices commonly used in model evaluation. The closer value to zero, the better model performance. 


$$
\text { RMSE }=\sqrt{\frac{\sum_{i=1}^{\mathrm{n}}\left(\mathbf{0}_{i}-\mathbf{P}\right)^{2}}{\mathrm{n}}}
$$

iii. Nasch and Sutcliffe Model coefficient efficiency (NSE): The NSE which compares the simulated values with the average observed is calculated from:

$$
\mathrm{NSE}=1-\frac{\sum_{1}^{n}\left(o_{i}-S_{i}\right)^{2}}{\sum_{1}^{n}\left(o_{i}-\bar{S}\right)^{2}}
$$

Where: $O_{i}$ is the observed value; $S_{i}$ is the simulated value; and $\bar{S}$ is the average value of observations. It ranges from $-\infty$ to 1 . When the value approaches to one, the prediction is better. The value of less than or equal to zero means that average values if used as predicted would be better than the model.

\section{Case study and application}

The intermittent instantaneous suspended sediment load and streamflow of Tigris River at Baghdad (Sarai station, latitude $33^{\circ} 18^{\prime} \mathrm{N}$, longitude $44^{\circ} 23^{\prime} \mathrm{E}$ ) operated by the Iraqi ministry of water resources are used in the study. The drainage area at this site is $134000 \mathrm{~km}^{2}$. The 112 daily flow and suspended sediment load data (1962 to 1981 water years) are used to train (calibrate) and testing (validation) the ANFIS and ANN models. The first 80 values were used for calibration and the remaining 32 for testing. Fig. 1 shows the locations of the Sarai sediment station at Baghdad.

Using the ANFIS model, different membership function was tried in order to find the optimum function that represent the observed suspended sediment load based on the assessment criteria ( $\mathrm{R}^{2}$, RMSE, and NSE). Thus, it was found that the triangular-shaped built-in membership function was the best representative of model prediction. For modelling the suspended sediment with the ANN's, all the data were standardized to be within $[-1,1]$.

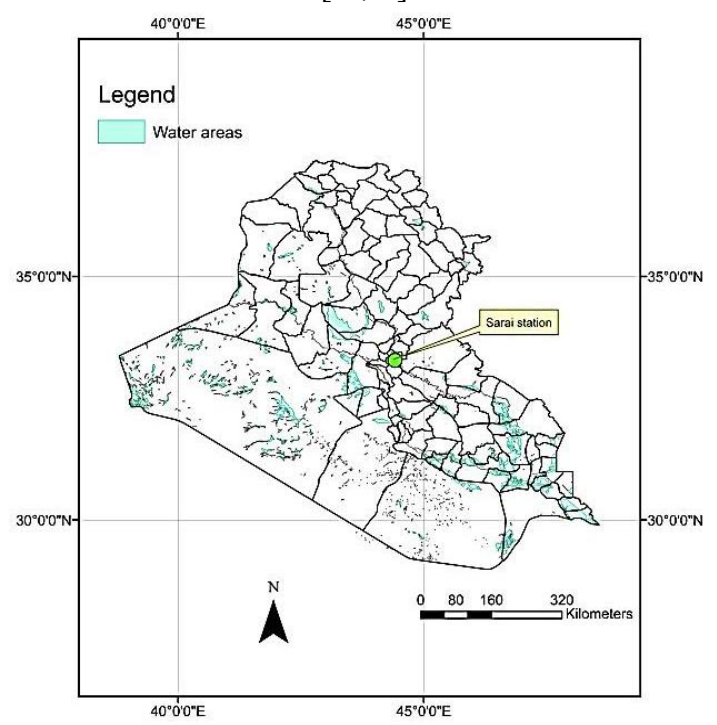

Fig. 1. The study area.

\section{Results and Discussion}

In each application of the evaluated methods, two data sets were used. The first was for the calibration and the second for the validation. In order to assess how well the methods performed, three evaluation criteria were used ( $\left.\mathrm{R}^{2}, \mathrm{RMSE}, \mathrm{NSE}\right)$. Table 1 shows the performance criteria of the evaluated methods. As it can be seen that $\mathrm{R}^{2}$, RMSE, and NSE of ANFIS were $0.58,75617$ and 0.58 , respectively during the calibration and 0.72 , 27944 , and 0.59 , respectively during the validation. While for the feedforward network were $0.55,101830$, and 0.54 , respectively during the calibration and 0.71 , 30395 , and 0.50 respectively during the validation. Using the radial basis network the values of $\mathrm{R}^{2}$, RMSE, and NSE were $0.64,89867$, and 0.64 , respectively during the calibration and $0.61,35190$, and 0.33 , respectively during the validation. The descending order of the performance was ANFIS, FFN, and RBN. Thus, it can be concluded that the ANFIS model outperform the other two evaluated methods. this could be attributed to the fact that the ANFIS model combines the linguistic representation of a fuzzy system with the learning ability of the ANN [20]. Thus, can be strongly eliminating pseudo signals (noise).

Figs. 2 and 3 show the observed suspended sediment load versus the modelled with the ANFIS model during the calibration and validation periods, respectively. As it can be seen that the modelled data were in a good agreement with the observed. Moreover, it can be noticed that the peaks values of observations during the both data sets (calibration and validation sets) were in a rather good consistence with those modelled. Figs. 4 and 5 show the observed and modelled suspended sediment load with the feed forward network during the calibration and validation, respectively. It can be seen that the performance during the calibration of the feedforward network was pretty well in matching the observed data set. While, however, during the validation there was a significant difference between the both data sets. As such it could be interpreted the lower values of $\mathrm{R}^{2}$, RMSE and NSE during the validation period with respect to ANFIS (Table 1). The feedforward network was not capable to model the peaks values of suspended load during the validation period. Peak values of sediment load are of significant importance in reservoir and dam management. Figs. 6 and 7 show the observed and modelled sediment load with the radial basis neural network during the calibration and validation, respectively. As with the feedforward, the radial basis network was pretty well capable to represent the observed sediment data during the calibration. While, during the validation, a divergence between the both data sets was observed. 


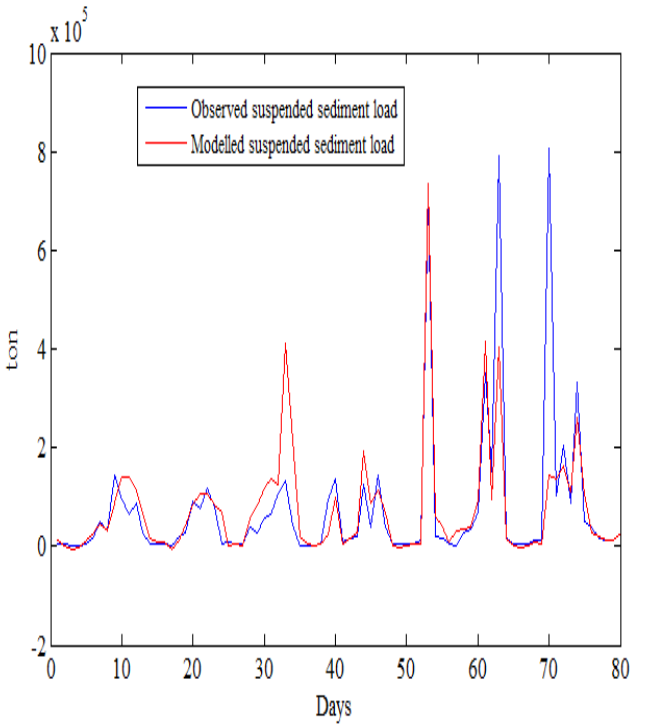

Fig. 2. Observed versus modelled suspended sediment load from ANFIS during the calibration.

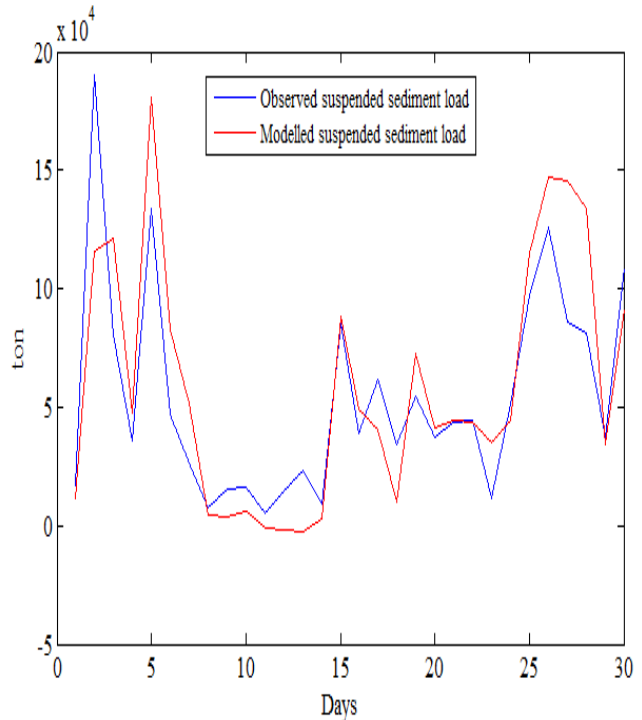

Fig. 3. Observed versus modelled suspended sediment load from ANFIS during the validation.

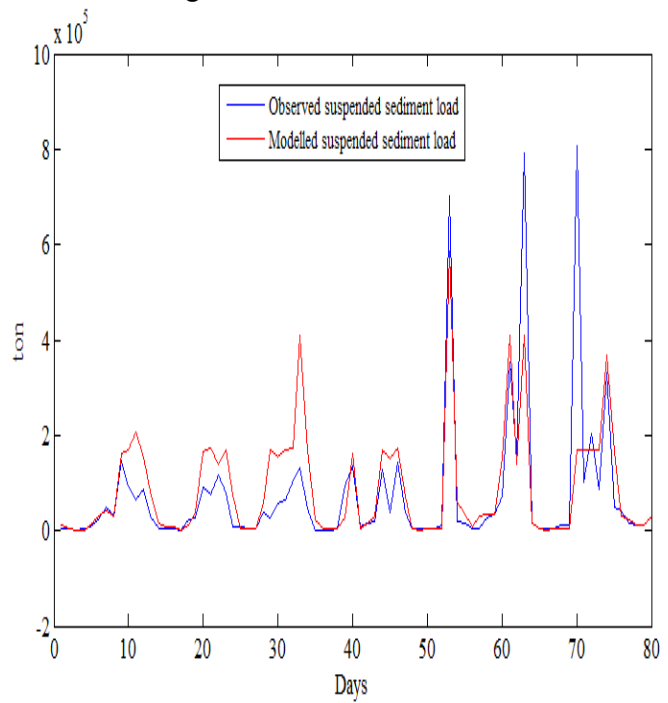

Fig. 4. Observed versus modelled suspended sediment load from feedforward network during the calibration.

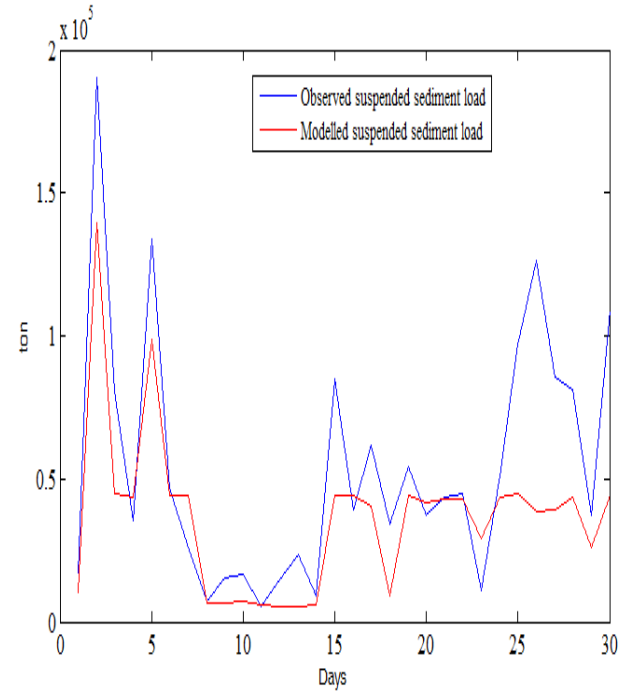

Fig. 5. Observed versus modelled suspended sediment load from feedforward network during the validation.

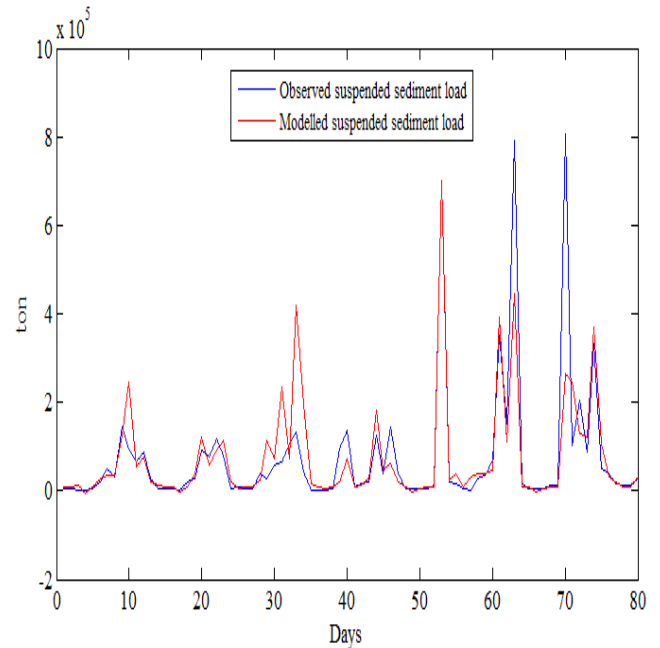

Fig. 6. Observed versus modelled suspended sediment load from radial basis network during the calibration.

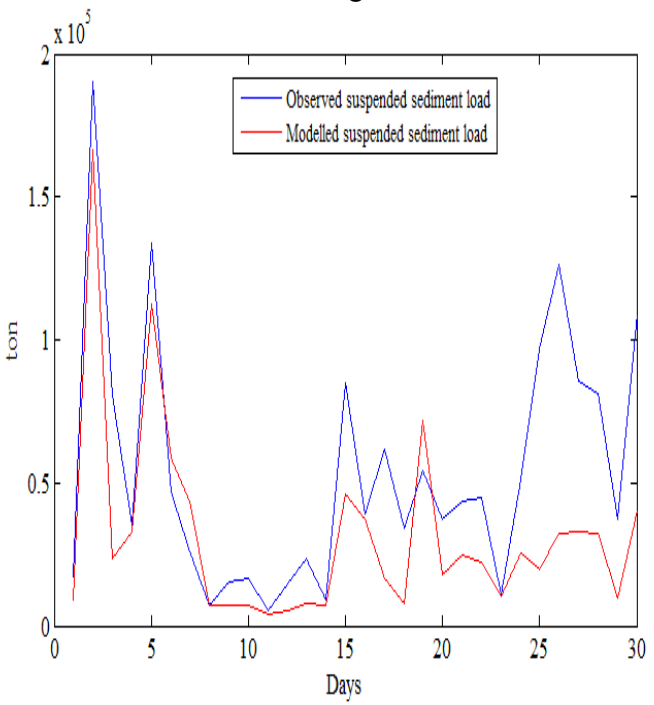

Fig. 7. Observed versus modelled suspended sediment load from radial basis network during the validation. 
Table 1. Performance criteria of the evaluated methods.

\begin{tabular}{|c|c|c|c|c|c|c|c|}
\hline \multirow{2}{*}{ Method } & \multicolumn{3}{|c|}{ Calibration } & & \multicolumn{3}{c|}{ Validation } \\
\cline { 2 - 8 } & $\mathrm{R}^{\wedge} 2$ & RMSE & NSE & & $R^{\wedge} 2$ & RMSE & NSE \\
\hline ANFIS & 0.58 & 75617.0 & 0.58 & & 0.72 & 27944.0 & 0.59 \\
\hline FFN & 0.55 & 101830.0 & 0.54 & & 0.71 & 30395.0 & 0.50 \\
\hline RBN & 0.64 & 89867.0 & 0.64 & & 0.61 & 35190.0 & 0.33 \\
\hline
\end{tabular}

\section{Conclusions}

This study evaluated three different types of artificial intelligence (ANFIS, FF and RB neural networks) to model the suspended sediment load of Tigris RiverSarai station in Baghdad. Three assessment criteria were used for the evaluation i.e. $\mathrm{R}^{2}$, RMSE and NSE. It was found that the ANFIS outperformed the other evaluated methods. This could be attributed to the ANFIS structure. The ANFIS integrates the advantage of the simplifying function of fuzzy reasoning and the self-learning ability of neural networks and thus gives a strong capability of eliminating noise [14]. This study could be of use to provide a better insight for the stake holders about the amount of suspended sediment load in Tigris River.

\section{References}

1. P. J. Wood and P. D. Armitage, Environ. Manage., 21, no. 2, pp. 203-217(1997).

2. J. Shiri and Ö. Kisi, J. Hydrol. Eng., 17, no. 9, pp. 986-1000 (2011).

3. B. Müller, J. Reinhardt, and M. T. Strickland, Springer Science \& Business Media (2012).

4. ASCE Task Committee on Application of Artificial Neural Networks, J. Hydrol. Eng., 5, no. 2, pp. 115-123 (2000a).

5. ASCE Task Committee on Application of Artificial Neural Networks, J. Hydrol. Eng., 5, no. 2, pp. 124-137 (2000b).

6. M. Alp and H. Cigizoglu, Environ. Model. Softw., 22, no. 1, pp. 2-13 (2007).
7. F. Anctil, M. Filion, and J. Tournebize, Ecol. Modell., 220, no. 6, pp. 879-887, ( 2009).

8. H. K. Cigizoglu and M. Alp, Adv. Eng. Softw., 37, no. 2, pp. 63-68, (2006).

9. M. Çimen, Hydrol. Sci. J., 53, no. 3, pp. 656-666, (2008).

10. E. Kakaei Lafdani, A. Moghaddam Nia, and A. Ahmadi, J. Hydrol., 478, pp. 50-62, (2013).

11. H. Kerem Cigizoglu and Ö. Kisi, J. Hydrol., 317, no. 3-4, pp. 221-238, (2006).

12. Ö. Kisi, Hydrol. Sci. J., 49, no. 6, pp. 3741,(2004).

13. Ö. Kisi and M. Zounemat-Kermani, Water Resour. Manag., 30, no. 11, pp. 3979-3994, (2016).

14. T. Rajaee, S. A. Mirbagheri, M. ZounematKermani, and V. Nourani, Sci. Total Environ., 407, no. 17, pp. 4916-4927, (2009).

15. Y.-M. Zhu, X. X. Lu, and Y. Zhou, Geomorphology, 84, no. 1-2, pp. 111-125, (2007).

16. J. J.-S. Roger, $A A A I$, , 91, pp. $762-767$ (1991).

17. A. Abraham, Fuzzy systems engineering, Springer, pp. 53-83 (2005).

18. S. Maiti and R. K. Tiwari, Environ. Earth Sci.,71, no. 7, pp. 3147-3160, (2014).

19. J. Si, Q. Feng, X. Wen, H. Xi, T. Yu, W. Li, and C. Zhao, J. Hydrol., 527, pp. 679-687, (2015).

20. M. Cobaner, B. Unal, and O. Kisi, J. Hydrol., 367, no. 1-2, pp. 52-61, (2009).

21. J. R. Jang, IEEE, 23, 3 (1993).

22. J.-S. R. Jang, C.-T. Sun, and E. Mizutani, (1997).

23. H. R. Maier and G. C. Dandy, Environ. Model. Softw., 15, pp. 101-124, (2000).

24. M. H. Beale, M. T. Hagen, and H. B. Demuth, "Neural Network Toolbox: users guide," (2012).

25. M. T. Hagan, H. B. Demuth, and M. H. Beale, Neural network design. (1996).

26. C. Santhi, J. G. Arnold, J. R. Williams, W. A. Dugas, R. Srinivasan, and L. M. Hauck, J. Am. Water Resour. Assoc., 37, no. 5, pp. 1169-1188, (2002)

27. Ö. Kisi, T. Haktanir, M. Ardiclioglu, O. Ozturk, E. Yalcin, and S. Uludag, Adv. Eng. Softw., 40, no. 6 , pp. 438-444, (2009). 